\title{
Contact Resistance Development of High Current Non-Overlapping Butt Contacts During a Large Number of Mechanical Switching Operations
}

\section{Conference Paper}

Author(s):

Menne, Henrik (D); Franck, Christian (D)

Publication date:

2019

Permanent link:

https://doi.org/10.3929/ethz-b-000388618

Rights / license:

In Copyright - Non-Commercial Use Permitted

Originally published in:

https://doi.org/10.1109/holm.2019.8923970 


\section{Contact Resistance Development of High Current Non-Overlapping Butt Contacts During a Large Number of Mechanical Switching Operations}

\author{
$1^{\text {st }}$ Henrik Menne \\ High Voltage Laboratory \\ ETH Zürich \\ Zürich, Switzerland \\ menneh@ethz.ch
}

\author{
$2^{\text {nd }}$ Christian M. Franck \\ High Voltage Laboratory \\ ETH Zürich \\ Zürich, Switzerland \\ franck@eeh.ee.ethz.ch
}

\begin{abstract}
Over the last years, the need for HVDC Circuit Breakers became obvious. The first HVDC grids are in operation and more grids are planned. To fully utilize the advantages of a grid, fast and reliable circuit breaker technology is needed. One technology that fulfills these requirements is the Hybrid HVDC Circuit Breaker. The key component of such a breaker is an Ultra Fast (mechanical) Disconnector (UFD). Such a disconnector must have a very short opening time and very low resistance in closed position. The goal of the present work is to develop a completely novel UFD platform. To minimize the opening time, non-overlapping butt contacts are used. Even though no fault current needs to be interrupted, the contact resistance will change during a large number of mechanical switching operations. An automatic test bench is developed to investigate this behavior. The first measurement investigates the influence of a high contact force on contact resistance. The second measurement determines the change of contact resistance during many operations. Both measurements are conducted with copper and chromium based materials. The performed measurements extend the knowledge of contact resistance to higher contact forces and develop an understanding of the change of contact resistance for nonoverlapping butt contacts without arcing. This will enable the dimensioning of high current contacts used in a UFD.

Index Terms - ultra fast disconnector, high voltage direct current, circuit breaker, high current, contacts, contact resistance, measurement setup
\end{abstract}

\section{INTRODUCTION}

In recent years, energy transmission over long distances has gained in importance [1], [2]. The increasing use of renewable energies in power generation and the long distance between these resources and consumers are the key factors behind this development. In order to decrease the losses, a high voltage is required. While this is possible in today's AC systems, the use of DC (HVDC) offers additional advantages. DC offers the possibility to use cables, because reactive power

This project is carried out within the frame of the Swiss Centre for Competence in Energy Research on the Future Swiss Electrical Infrastructure (SCCER-FURIES) with the financial support of the Swiss Innovation Agency (Innosuisse - SCCER program)

This work is supported by Plansee, who provided the raw material for the contacts.

We would like to thank Fabian Mächler for his help in building and setting up the test bench. losses are negligible. Furthermore, the active power losses are smaller and the power flow is fully controllable [3]. While the ability to control the power flow is also true for point-to-point connections, the full potential is only achieved with a grid. This increases the reliability and offers more flexibility, but a grid can only be operated with circuit breakers (CB). A CB can switch load and fault currents and enables the isolation of a fault and thus a reconfiguration of the grid. In contrast to an AC-grid, the peak fault current is not limited by the reactance of the grid, but only by the small DC-resistance. However, the rate-of-rise of the fault current is limited by the line inductance. An additional inductance can be used to limit the rate-of-rise, but a larger inductance stores more magnetic energy that the $\mathrm{CB}$ has to dissipate [4]. To limit the stress for all components, a fast $\mathrm{CB}$ is needed. One technology that fulfills the technical requirements is the Hybrid-HVDC CB [5]. The CB consists of three parallel paths, which are depicted in Fig. 1. During normal operation, only the nominal current path is switched on. This path includes a load commutation switch (LCS) and an Ultra Fast Disconnector (UFD). The LCS is usually a semiconductor device and therefore operates faster than a mechanical operated switch. The resistance during on-state can be in the range of a few milli ohm, which is in the same order of magnitude as the contact resistance of the investigated contacts after more than thousand mechanical switching operations. In case a fault is detected, the main breaker path is switched on and the LCS off. Therefore, the current will commutate. Now the UFD can open without the need to break the fault current. The main breaker is made up of a few hundred semiconductor (SC) devices and can switch the current off. The remaining energy of the line is dissipated as heat in the energy absorbing path. Usually metal oxide varistors (MOV) are used in this path.

In a Hybrid-HVDC $\mathrm{CB}$, the internal current commutation time is dominated by the operating time of the UFD [6]. As a result optimizing the switching time of the UFD plays a major role in the development of a Hybrid-HVDC CB. A couple of requirements, which are sometimes contradictory, influence the switching time. The main parameters that have an influence 


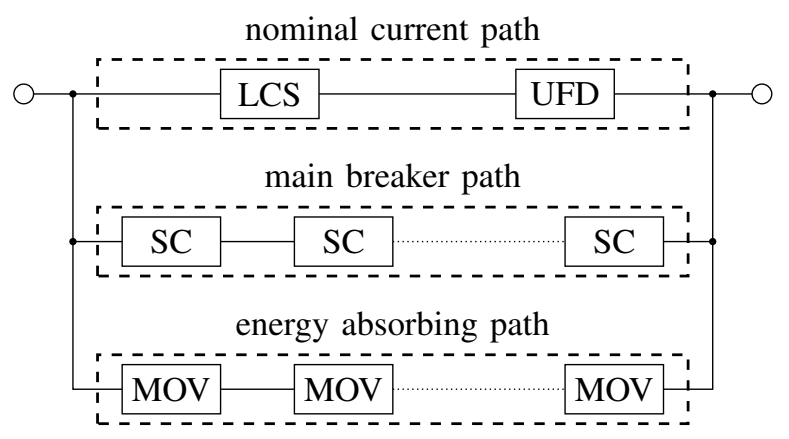

Fig. 1. Schematic of a Hybrid-HVDC CB

on opening time are the contact force and the required gap distance to withstand the transient interruption voltage (TIV). A higher contact force provides a lower contact resistance and therefore lower losses and heating in the contact, but it will increase the opening time. The dependency between contact force and contact resistance is part of the investigation that is shown in this paper. A smaller contact gap leads directly to a shorter opening time, but the electric stress over the open contacts is higher. Therefore, a smooth contact surface is needed. This leads to a special contact design, which is presented in detail in Section III-A. The different aspects in the design are elaborated in detail in [7].

Literature suggests that the relation between contact force and resistance can be described with Equation (1). The value of the exponent is not a constant and can vary. Vinaricky [8] lists different publications which show an exponent in the range from -0.3 to -3 . Most papers seem to find an exponent in the range from -0.9 to -1 for pure metal. It is notable that many publications use a contact force less than $10 \mathrm{~N}$ and a half sphere contact with a diameter of a few millimeters. The measurements presented in this paper have been carried out with a contact force up to $10 \mathrm{kN}$ and use contacts with diameters greater than $50 \mathrm{~mm}$.

$$
\frac{R_{\mathrm{c}}}{1 \Omega}=\left(\frac{F}{1 \mathrm{~N}}\right)^{x}, \quad x \approx(-0.9) \ldots(-1)
$$

Some literature [9] suggest that a contact behaves differently with a contamination layer, of Equation (2). The factors $a_{\text {metal }}$ and $a_{\text {cont }}$ are in such way, that for low forces the contamination part $\left(F^{-1}\right)$ dominates. The result of Equation (2) for an increasing force is that the contact resistance decreases for low forces faster than for large forces.

$$
R_{\mathrm{c}}=a_{\mathrm{metal}} F^{-0.5}+a_{\mathrm{cont}} F^{-1},
$$

The previous paper [7] showed the measurement setup in detail and presented first results. The new measurements presented in this paper are based on an extended version of the used test setup, which is presented in Section II.

The main scope of this paper is to extend the known relationship between contact force and contact resistance to a higher contact force, larger contact area and to develop an understanding of the change of contact resistance after a large number of mechanical switching operations. Mechanical switching operation in this paper refers to opening or closing the contacts without current flow and thus without current interruption.

\section{Measurement Setup}

The schematic of the measurement setup is depicted in Fig. 2. The setup consists of a direct current source, a voltage measurement device and a test bench to press two contacts with a specified force together. The direct current source is composed of four Elektro Automatik PSI 9080-510 power supplies. Each can supply up to $510 \mathrm{~A}, 80 \mathrm{~V}$ or $15 \mathrm{~kW}$, depending on the mode of operation. The power supplies are connected in parallel and deliver up to $2040 \mathrm{~A}$. The voltage measurement device is a Labjack 77 Pro. It has four different voltage ranges $(0.01 \mathrm{~V}, 0.1 \mathrm{~V}, 1 \mathrm{~V}$ and $10 \mathrm{~V})$ and an effective resolution of up to 21.8 bit. The device measures the contact voltage, the voltage of the load cell and the thermoelectric voltage of one Type $\mathrm{T}$ thermocouple, which is attached to the lower contact. Furthermore, a digital sensor (EI1050) is connected to the Labjack to measure the ambient temperature and relative humidity. A HBM U10M (load cell) in combination with a $H B M$ MC3 (signal converter) is used to measure the applied force directly.

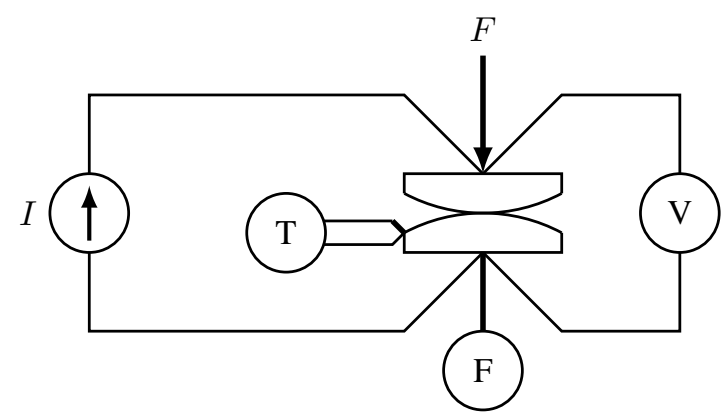

Fig. 2. Schematic of Measurement

A stepper motor is used to move the contacts and generate the contact force. This configuration allows applying a contact force between $10 \mathrm{~N}$ and $10 \mathrm{kN}$.

The control of the measurement setup and logging of the data is realized with a python program. Contact voltage and measurement current are measured in a $1 \mathrm{~s}$, contact force in a $0.5 \mathrm{~s}$ and temperatures and relative humidity in a $10 \mathrm{~s}$ interval.

\section{CONTACT MATERIAL}

The measurements that are presented in this paper have been carried out with three materials:

- copper - $\mathrm{Cu}$

- copper with $2 \%$ of the total mass chromium - $\mathrm{CuCr} 2$

- copper with $25 \%$ of the total mass chromium - $\mathrm{CuCr} 25$

The choice of material is of great significance. For most AC CB or disconnectors the best material is well known and specific to the type of switchgear. As an example: the preferred 
material for a vacuum interrupter is copper chromium and the arcing contacts in a $\mathrm{SF}_{6} \mathrm{CB}$ are made from tungsten copper.

However, the optimal material for an UFD has not been found yet. The required properties are quite different to those of an AC CB or other known switchgear. The current is commutated with the LCS; this means the contacts are not exposed to arcing, so the resistance against contact erosion or the chopping current of the material is not critical. The proposed contacts use a special version of butt contacts and are pressed together with a specific contact force. This configuration is similar to a vacuum interrupter.

One possible material is copper, it is cheap and the conductivity is very good. Nevertheless, the hardness of the material is relatively low and it oxidizes in air. This material is considered as a baseline. The second and third material that were chosen are sintered materials, with a mass percentage of chromium of $2 \%$ and $25 \%$. Chromium is lighter (a lighter contact is faster to accelerate) than copper but also harder. On the other hand, the conductivity is significantly lower. A comparison between the materials is given in table I.

TABLE I

COMPARISON BETWEEN THE TESTED MATERIALS AND CHROMIUM [9], [10]

\begin{tabular}{lcccc}
\hline material & $\mathrm{Cu}$ & $\mathrm{Cr}$ & $\mathrm{CuCr} 2$ & $\mathrm{CuCr} 25$ \\
\hline copper volume in \% & 100 & 0 & 97.6 & 71.3 \\
chromium volume in $\%$ & 0 & 100 & 2.4 & 29.4 \\
specific conductivity & 54.7 & 8 & 48 & 31 \\
in $10^{6} \mathrm{~S} \mathrm{~m}^{-1}$ & 8.96 & 7.19 & 8.7 & 8.05 \\
density in kg m$^{-3}$ & $85-120$ & $120-570$ & 110 & 70 \\
hardness HV & & & & \\
\hline
\end{tabular}

\section{A. contact shape}

The used contact shape is derived from the Rogowski profile, which is a common choice if a homogeneous electric field between two electrodes is needed [11]. However, this shape is not designed to make the best contact and therefore to carry a large current. The profile is chosen because a homogeneous electric field between the separated contacts minimizes the required gap distance and therefore the time, which is needed to withstand the TIV.

The Rogowski profile is described by Equation (3). The parameter $A$ is the characteristic distance between the Rogowski profile and an infinite ground plane. With variable $t$ the development of the profile is determined.

$$
\begin{aligned}
& x=\frac{A}{\pi} \cdot t \\
& y=\frac{A}{\pi} \cdot\left(\frac{\pi}{2}+\mathrm{e}^{t}\right)
\end{aligned}
$$

In Fig. 3 the cross-section of the used contacts is depicted. The used parameters are: $A=5 \mathrm{~mm},-4.8 \pi \leq t \leq 1.5$.

\section{MEASUREMENTS}

The measurements are split into two parts: the preparation of the contacts and the measurement itself.

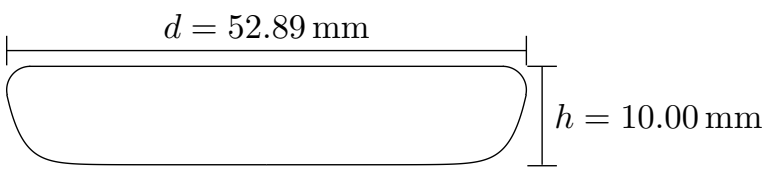

Fig. 3. Cross-section of the used contacts with a Rogowski profile

\section{A. contact preparation}

In order to receive the best possible results, a specified procedure is conducted before each experiment. The contacts are cleaned with hydrochloric acid $(\mathrm{HCl}, 4 \%)$. $\mathrm{HCl}$ removes the oxide layer of copper. It also removes the oxide layer of chromium, but also dissolves chromium. Therefore, it is important to keep the time in $\mathrm{HCl}$ bath as short as possible. The oxide layer is removed within seconds. After that, sandpaper (grit designation: P280) is used to roughen the contacts. No special grinding pattern is applied. Finally, the contacts are cleaned with ethanol. This procedure is carried out before each measurement series, so that each series has the same starting conditions.

\section{B. contact resistance as a function of contact force}

To determine the dependency between contact resistance and contact force, an increasing force is applied. Each measurement series starts with $10 \mathrm{~N}$ and ends with $10 \mathrm{kN}$. Care is taken to ensure that during the closing of the contacts the contact force never exceeds $10 \mathrm{~N}$. The same contacts can be used again, if they are prepared according to the procedure described in Section IV-A. From literature it is known that the contact resistance should be inversely-proportional to the contact force. Therefore, the decision is made to apply the force in 19 logarithmic spaced levels. With each material three measurement series are conducted. During two series each level of the contact force is applied for $7 \mathrm{~min}$ and during one series for $45 \mathrm{~min}$. One example for the measured force is depicted in Fig. 4. The signal looks noisy for small forces, but this is mainly an effect of the logarithmic y-axis.

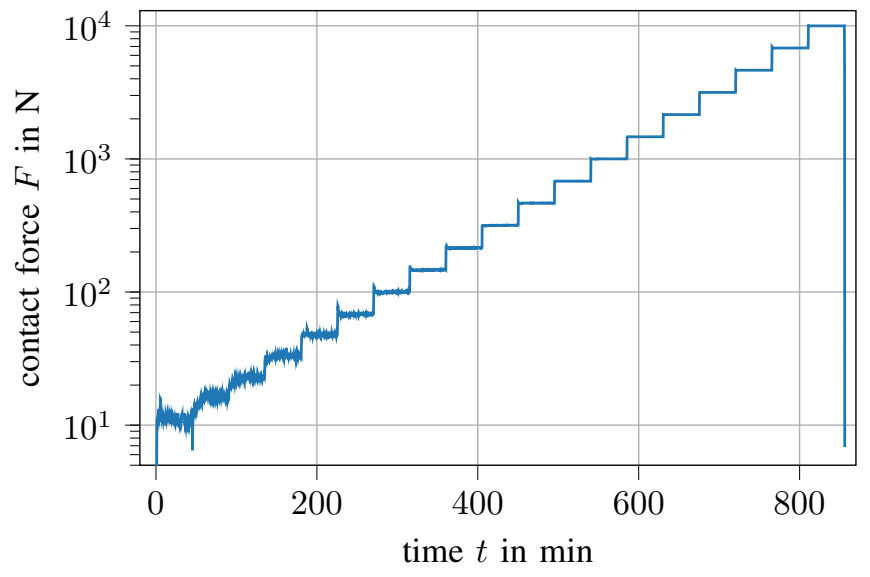

Fig. 4. Applied contact force during one measurement series to determine the relationship between contact resistance and contact force 
The choice of the measurement current directly influences the measurement results. A low current results in small signal which is hard to measure, but it limits the generated heat in the contacts. A significant increase of the contact temperature has to be avoided, because it can influence the measurement results. During first tests it was found that a measurement current of $100 \mathrm{~A}$ is large enough to generate a voltage drop that is for any measured resistance greater than $50 \mu \mathrm{V}$, which is well above the resolution of the used data acquisition unit. Additionally the current is low enough that the contacts do not heat up significantly.

During the test procedure the contact force is only increased, no measurements are taken during the decrease of the contact force. The contact resistance is always lower if the contacts have seen a higher contact force before. The reason for this behavior is not investigated in this paper, but it is very likely that once a good contact is made (high contact force), it will hold a part of the contact while the force is decreasing.

The contact resistance is calculated with Equation (4). The analytical calculation of the bulk resistance $R_{\text {bulk }}$ of a complex shape, like the used Rogowski shaped contacts, is not straightforward and not performed in this paper. A simulation was performed to determine the bulk resistance $R_{\text {bulk }}$ of one contact at room temperature. It depends on the material $M$ and the contact temperature $T$ and is in the range of a few hundred nano ohm. As the temperature increases only a few kelvin, the temperature dependency of the material can be assumed linear. The bulk resistance is calculated for each resistance value with the measured temperature.

$$
R_{\mathrm{c}}=\frac{V}{I}-R_{\mathrm{bulk}}(M, T)
$$

As described in Section I, the relationship between contact resistance and contact force changes if a contamination layer is present. Therefore, two power law functions are used to characterize the behavior. For each force level the mean contact resistance and the mean measured force are calculated. Then an optimizing algorithm is used to split this dataset, with respect to force, into two parts, so that the sum of the residuals of the fitted power law functions is minimal.

\section{C. contact resistance as a function of mechanical switching}

The second type of measurement series in this paper is carried out to investigate the change of contact resistance during a large number of mechanical switching operations. Since neither a standard for HVDC switchgear nor practical experience exists, the requirements for an $\mathrm{AC} \mathrm{CB}$ are used. The standard IEC 62271:100 specifies at least 2000 mechanical operations for high voltage (AC) circuit breaker of the M1 class [12]. Class M1 is used for standard CB with normal mechanical endurance.

During this measurement, a constant force $(F=400 \mathrm{~N})$ is applied during each operating sequence. The chosen force is just an example without a specific background. It is assumed that the foreseen actuator can produce a contact force in this order of magnitude. The measurement current is 100 A. During each sequence, the force is applied for $90 \mathrm{~s}$. The contacts are then separated and closed again. Before the contacts are opened, the current is switched off. Measurement series with 1000 and 2500 operations are performed. The mean contact resistance of each operation is calculated and used in the later evaluation.

\section{D. measurement period}

The contact resistance decreases with time, even if the contact force is constant. This is well known in literature and explained by a growing area of electrical contact under constant load [9]. The largest change of the contact resistance can be seen within the first minutes after the contacts have been closed. An example is given in Fig. 5. After $20 \mathrm{~min}$, the resistance is about $8 \%$ less than at the beginning of the measurement and has not reached a steady state. Other measurements show that it can take up to one hour until a steady state is reached, which is in good agreement with literature [13]. As all operations show a similar decrease and it is not feasible to conduct many measurement series if one measurement point takes longer than $20 \mathrm{~min}$, the decision is made to perform only short measurements and not to compare the static value of the contact resistance. As the results will show, the observed changes are much larger than this change of resistance.

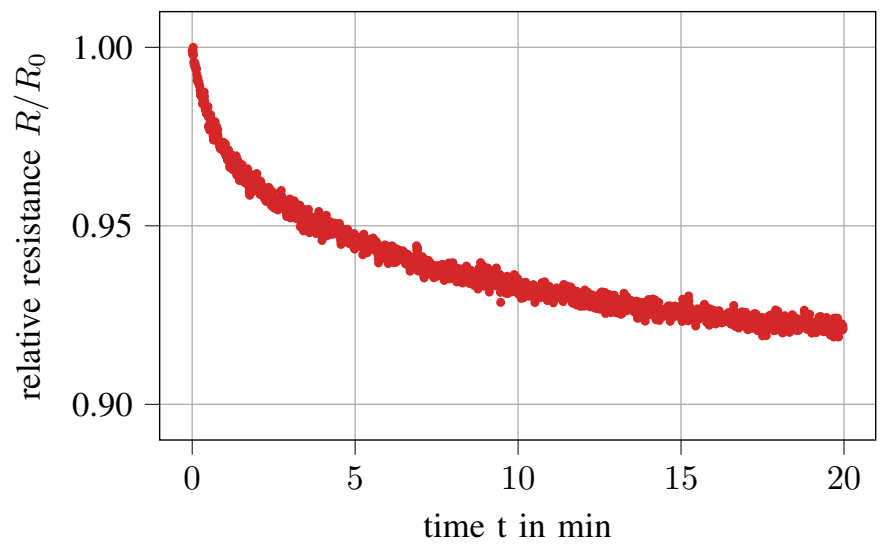

Fig. 5. Change of the contact resistance over a period of $20 \mathrm{~min}$ with a constant force $F=400 \mathrm{~N}$, the resistance is normalized to first measurements

\section{E. low contact force}

The test bench used in this paper is optimized to apply a force between $100 \mathrm{~N}$ and $1000 \mathrm{~N}$. This range is extended $(10 \mathrm{~N}-10 \mathrm{kN})$ to get a better understanding of the relationship between contact force and contact resistance. The difference between measured force and set force is limited to $2 \mathrm{~N}$ or $0.1 \%$ of the set force, whichever is greater. Therefore the variance, seen in the force measurement (Fig. 4), is greater for low forces. 


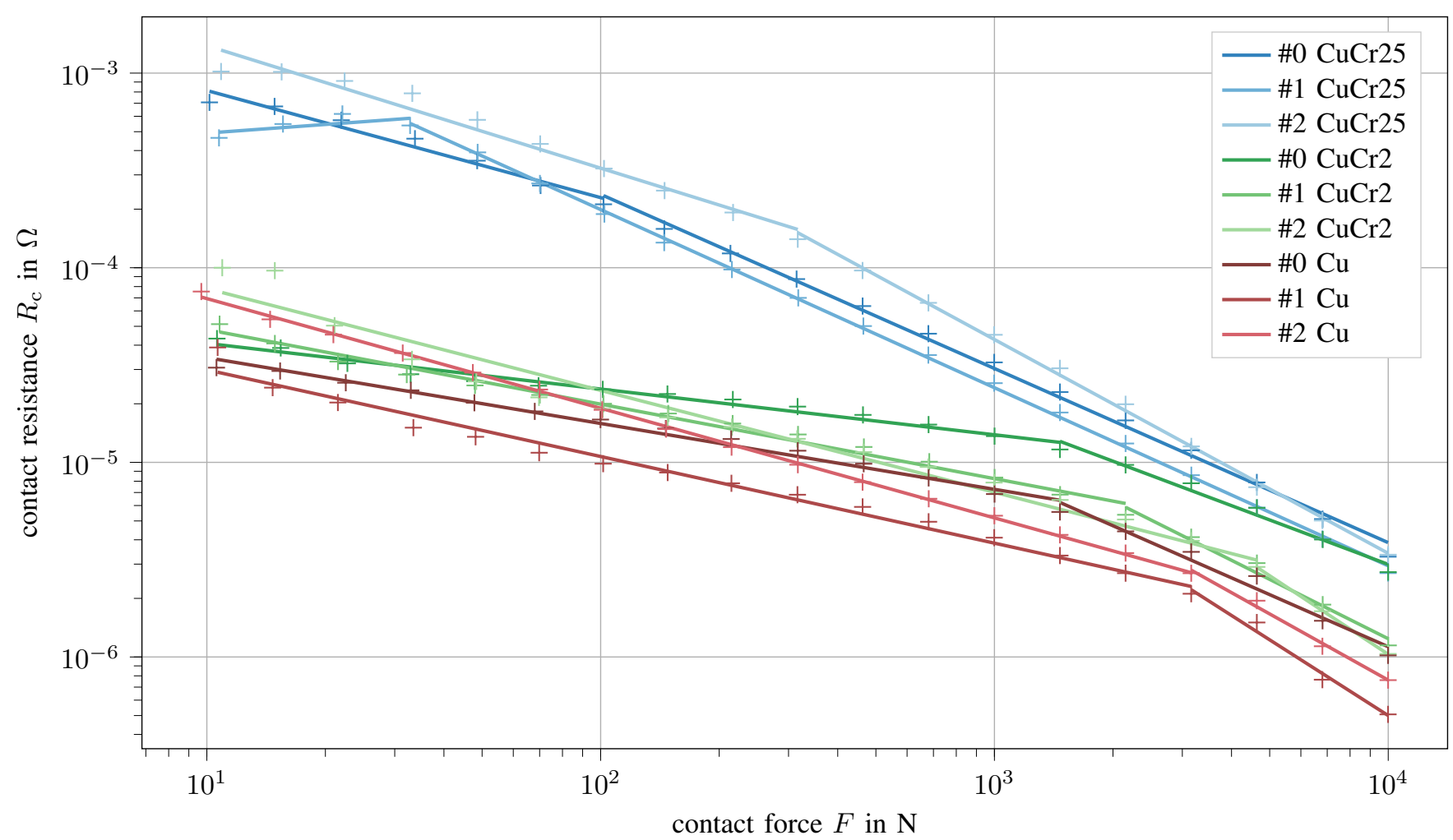

Fig. 6. Contact resistance in dependence of contact force, comparison between $\mathrm{Cu}, \mathrm{CuCr} 2$ and $\mathrm{CuCr} 25$, the markers show the measured contact resistance, the lines are two fitted power law functions, as described in Section IV-B

\section{RESULTS}

The results from all measurements and with all three contact materials are reported in this section.

\section{A. contact resistance as a function of contact force}

The measured contact resistances with varying contact forces according to the procedure described above in Section IV are shown in Fig. 6. For each material, three different measurement series were performed. The ones labelled with \#0 denote the series with $45 \mathrm{~min}$ duration on each force level. Measurement series marked with \#1 and \#2 are executed with $7 \mathrm{~min}$ on each force level. The experiment series with the same material are conducted with the same contacts, which are prepared according to the procedure described in Section IV-A. No systematic difference can be observed in the results with the same contact material but different duration on each force level. In addition, the three series for each material indicate a good reproducibility of the measurements.

From Fig. 6 it can also be seen that pure $\mathrm{Cu}$ and $\mathrm{CuCr} 2$ behave quantitatively and qualitatively very similarly. The measured contact resistance is in the order of $10 \mu \Omega$ to $100 \mu \Omega$ at low contact forces below $100 \mathrm{~N}$ and decreases to values around $1 \mu \Omega$ at higher forces around $10 \mathrm{kN}$. As described above, the results are fitted with two power law functions. The resulting exponents and the force value at which the transition takes place are shown in Table II. For both materials and all measurement series, the transition is observed in the range of $1 \mathrm{kN}$ to $5 \mathrm{kN}$. The decrease of contact resistance with increasing force is steeper in the higher force range.

TABLE II

EXPONENT FOR THE FITTING FUNCTION

\begin{tabular}{llrrr}
\hline & & curve I & curve II & Transition \\
\hline \multirow{3}{*}{$\mathrm{Cu}$} & $\# 0$ & -0.34 & -0.89 & $1.5 \mathrm{kN}$ \\
& $\# 1$ & -0.45 & -1.29 & $3.2 \mathrm{kN}$ \\
& $\# 2$ & -0.56 & -1.13 & $3.2 \mathrm{kN}$ \\
\hline \multirow{3}{*}{$\mathrm{CuCr} 2$} & $\# 0$ & -0.23 & -0.76 & $1.5 \mathrm{kN}$ \\
& $\# 1$ & -0.38 & -1.01 & $2.2 \mathrm{kN}$ \\
& $\# 2$ & -0.52 & -1.34 & $4.6 \mathrm{kN}$ \\
\hline \multirow{3}{*}{$\mathrm{CuCr} 25$} & $\# 0$ & -0.55 & -0.89 & $102 \mathrm{~N}$ \\
& $\# 1$ & 0.15 & -0.92 & $33 \mathrm{~N}$ \\
& $\# 2$ & -0.63 & -1.10 & $317 \mathrm{~N}$ \\
\hline
\end{tabular}

The contact resistances with $\mathrm{CuCr} 25$, in turn, behave qualitatively and quantitatively different. Firstly, it is notable that the contact resistances at lower forces are around one order of magnitude higher than for the two other materials. In addition, the transition between the two power law curves takes place at considerably lower forces of $30 \mathrm{~N}$ to $300 \mathrm{~N}$. This leads to the situation that for forces above approximately $1 \mathrm{kN}$, the contact resistances of all three materials are in the same order of magnitude.

Due to the experimental setup, the deviation of the measured force from the set force can be higher for low forces (Section IV-E). This leads to a higher variance in the measured 


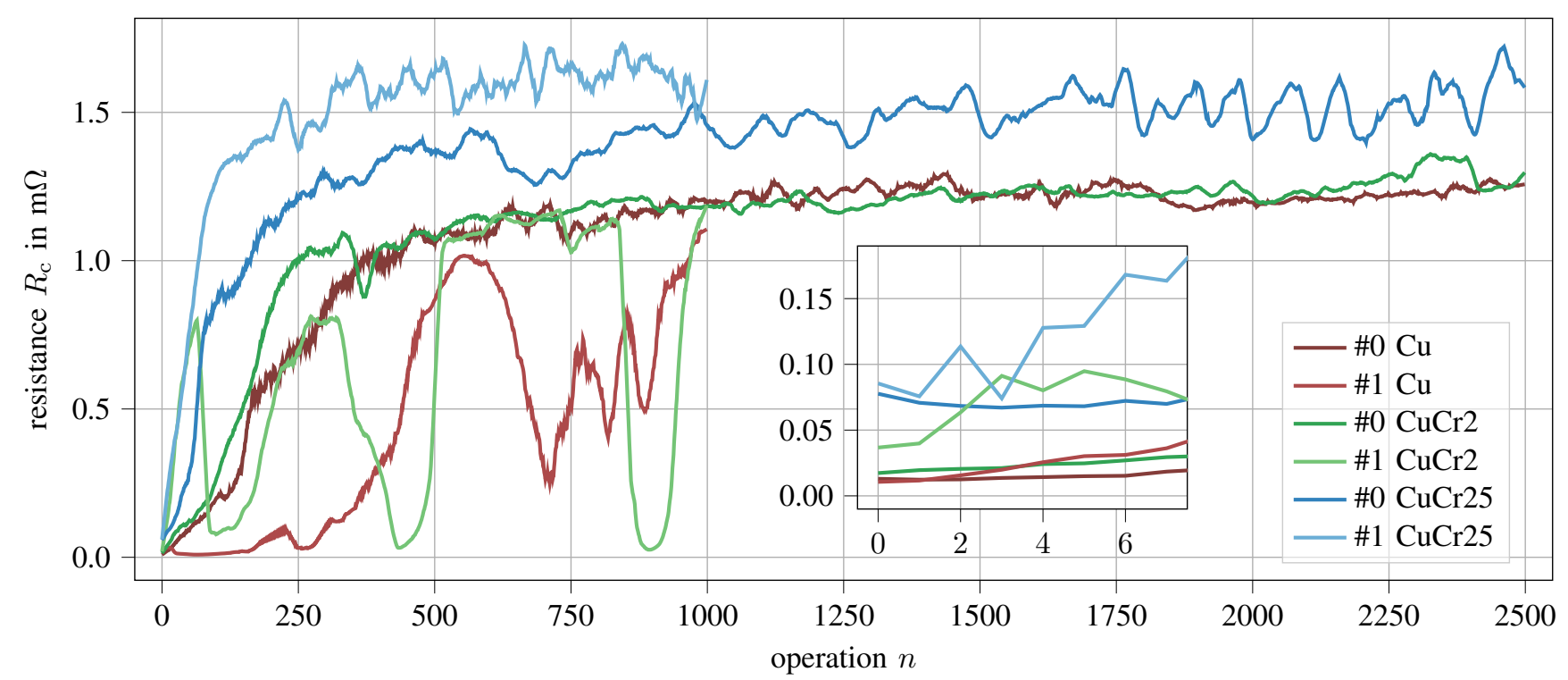

Fig. 7. Resistance change during a large number of mechanical switching operations, the contact resistance is filtered with a Savitzky Golay filter (window: 21 operations, polynomial order: 1); data in zoomed view is not filtered

resistance. The observed variance is small compared to the changes caused by the change of contact force.

\section{B. contact resistance in dependency of cycles}

The measured resistance during a large number of mechanical switching operations is depicted in Fig. 7. The series denoted with \#0 contain 2500 mechanical switching operations and the ones denoted with \#1 1000. All series have in common that the resistance is low for the first few operations (as can be seen in the zoomed section) and then increases.

The contact resistance of the first operation is in a similar range for all materials when compared to Fig. 6. This means that the contact resistance of $\mathrm{CuCr} 25$ is significantly higher than of $\mathrm{Cu}$ or $\mathrm{CuCr} 2$, which are relatively close to each other (Table III). The change of contact resistance during the mechanical switching operations is not similar for all measurement series. For some series the resistance increases very fast (e.g. \#1 $\mathrm{CuCr} 25)$, other measurement series show an almost constant resistance for the first ten or hundred operations (e.g. \#1 Cu). It is common for all series that resistance rises at some point and the series with 2500 operations show that it can reach a steady value. The change of resistance between first operation and steady state value is arbitrary and not reproducible. The performed measurements show that the change of resistance is not monotonic, even if the values are smoothed with a Savitzky Golay filter. If the measurement data is not smoothed, the arbitrary character of the contact resistance is sometimes even more pronounced. One example for such a series is $\# 1 \mathrm{Cu}$, which is depicted in Fig. 8.

The resistance of the first operation and the steady state value of the measurement series denoted with \#0 (2500 operations) are shown in Table III. The change of resistance during at least one hundred operations has to be very small to calculate the steady state contact resistance, therefore it is not

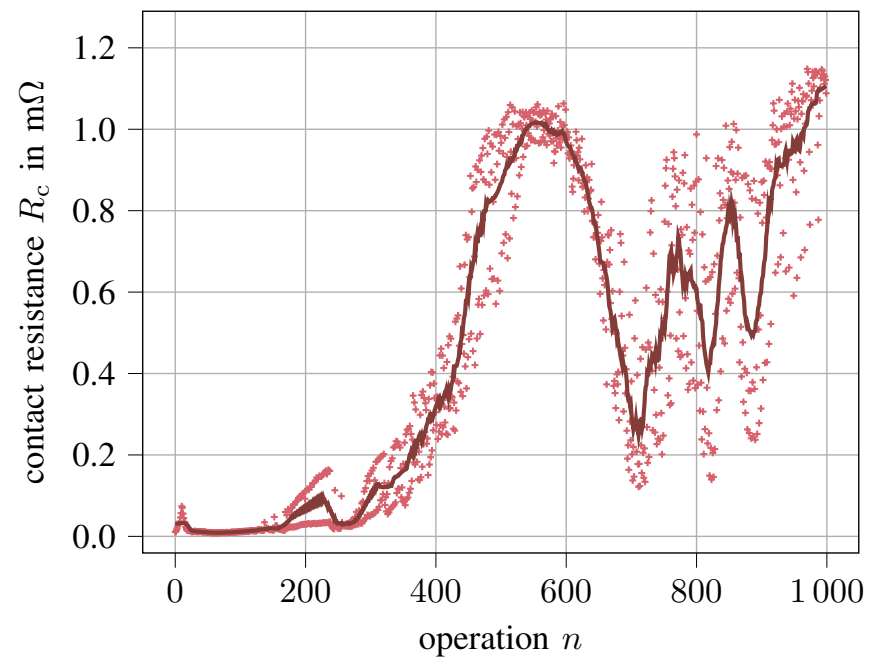

Fig. 8. Non-monotonic behavior of contact resistance with scattering, \#1 Cu

reasonable to calculate such a value for a measurement series like \#0 Cu. In this paper the steady state value is calculated, as the mean value of the last 1000 operations. It can be seen that the difference between the materials is decreasing. During first operation the resistance of $\mathrm{CuCr} 2$ is around $34 \%$ higher compared with $\mathrm{Cu}$; over the last 1000 operations it is only $2 \%$ higher. This behavior is even more emphasized for $\mathrm{CuCr} 25$. The first operation shows a resistance which is almost six times larger than the corresponding $\mathrm{Cu}$ resistance. After 1000 operations $\mathrm{CuCr} 25$ has reached a stable value and is oscillating around this value. The mean value over the last 1000 operations is only $25.5 \%$ higher.

The measured values scatter around the smoothed line. 
TABLE III

DIFFERENCE OF THE CONTACT RESISTANCE BETWEEN CU, CUCR2 AND CUCR25 AT THE BEGINNING OF A MEASUREMENT SERIES $R_{\mathrm{C}, 0}$ AND AFTER 2500 MECHANICAL SWITCHING OPERATIONS $R_{\mathrm{C} \text {,end }}$ (ARITHMETIC MEAN OVER THE LAST 1000 OPERATIONS)

\begin{tabular}{llrrr}
\hline & \multicolumn{2}{c}{$R_{\mathrm{c}, 0}$} & \multicolumn{2}{c}{$R_{\mathrm{c}, \text { end }}$} \\
material & in $\mu \Omega$ & in $\%$ & in $\mathrm{m} \Omega$ & in $\%$ \\
\hline $\mathrm{Cu}$ & 13.0 & 100.0 & 1.22 & 100.0 \\
$\mathrm{CuCr} 2$ & 17.3 & 133.6 & 1.25 & 102.3 \\
$\mathrm{CuCr} 25$ & 77.6 & 596.9 & 1.53 & 125.4 \\
\hline
\end{tabular}

This scattering is changing over the measurement. It is less pronounced between operation 600 and 700 and more between operation 700 and 900 . The resistance change of two consecutive measurements during this particular measurement is up to a factor of 6 . The change of resistance between two consecutive measurements has to be taken into account when selecting the voltage range of the measurement device for the next operation. The used measurement setup is capable of selecting the optimal voltage range for the next operation based on the measurements of the previous one. Not all measurement series show such a distinctive scattering. One series with little scattering is \#1 CuCr2 and is depicted in Fig. 9.

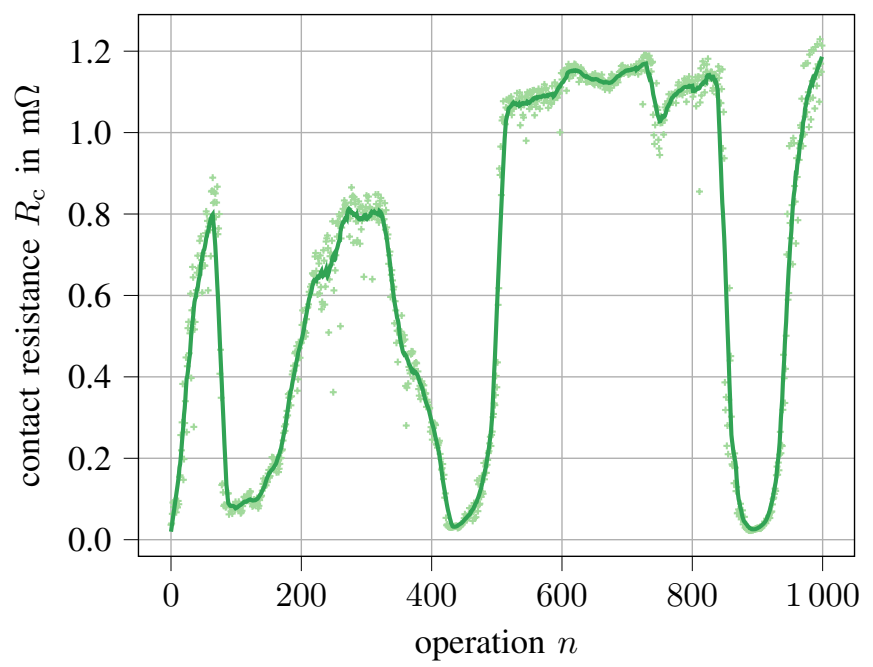

Fig. 9. Non monotonic behavior of contact resistance with little scattering, \#1 CuCr2

The overall development of the contact resistance is similar. Both, $\mathrm{Cu}$ and $\mathrm{CuCr} 2$, start with low resistance, which increases and decreases during the measurement a few times. But the scattering of the measurements around the smoothed line is different. Only a few values show a large deviation from the smoothed line; most of the values are at or in the vicinity of it.

\section{DISCUSSION}

The goal of this investigation was to get a better understanding of the development of contact resistance during a large number of mechanical switching operations. In order to achieve this goal, the trend of the contact resistance with a constant force was studied. The results show that once the contact is made contact resistance drops with time. It is found that for the given contact shape after $20 \mathrm{~min}$ to $45 \mathrm{~min}$ an almost stable resistance value is achieved. The resistance drop occurs with all tested materials and is usually less than $10 \%$. A direct comparison with literature is not possible, since the time until a steady state resistance is reached depends on the contact size and shape. In [9] is reported that small contacts may need only a few seconds, but big contacts may need more then half an hour. Shea [13] mentions that the resistance of a molded case circuit breaker took $45 \mathrm{~min}$ to stabilize. Therefore, it can be stated that the time until a stable resistance was reached is in the range of what is known from literature. It is also in good agreement with literature that the contact resistance, once a contact is made, decreases over time and never increases. The drop of contact resistance is well below the change of contact resistance that can be observed during the contact force as a function of contact force measurement (Section V-A). Therefore, the resistance drop over time has no influence on the overall trend in the conducted measurements, which allows a shorter measurement duration per operation

The contact resistance varies between two consecutive measurement series, even if the contact preparation was the same. This is in good agreement with literature, where it is shown that the contact resistance scatters. On a microscopic level the contact alignment may be different for the same force, and therefore the resistance is different. This scattering is independent of the contact force.

The relationship between contact force and contact resistance is defined by two power law functions. Contradictory to known literature the steeper drop of resistance occurs at higher forces. The literature shows that a steeper drop is observed with a contamination layer. Once the contamination layer is broken, the pure metal to metal contact determines the steepness, which is usually lower. However, this explanation cannot be applied to the results in this paper. All tested materials show a slower decrease of contact resistance with lower forces than with higher forces. Physically it does not make sense that the contamination layer is only present for high forces. However, the applied forces during the investigations are orders of magnitudes higher than in the known publications, therefore, it could be possible that a third behavior is observed. During this regime the slope is in a similar range compared to the regime that occurs with a contamination layer. Until now it is not clear what causes the behavior. To investigate it in more detail the range of applied forces should be extend to lower forces (known from literature), so that all three regimes can be observed in one measurement.

The analysis of the relationship between contact force and contact resistance shows that $\mathrm{Cu}$ and $\mathrm{CuCr} 2$ behave similarly. On the one hand this can be expected, because both materials consist mainly of copper. On the other hand the manufacturing process is different for both. $\mathrm{Cu}$ is cast and then hot pressed; $\mathrm{CuCr} 2$ is a sintered material. As a result, $\mathrm{Cu}$ is usually softer. The difference between $\mathrm{Cu} / \mathrm{CuCr} 2$ and $\mathrm{CuCr} 25$ is distinctive. 
In general, $\mathrm{CuCr} 25$ has a higher contact resistance. This is in particular true for contact forces below $1 \mathrm{kN}$, where the resistance is up to one order of magnitude higher. Above this force the difference is smaller but the resistance remains higher than $\mathrm{Cu} / \mathrm{CuCr} 2$. As mentioned before, two power law functions can describe the relation. The transition between them occurs for $\mathrm{Cu}$ and $\mathrm{CuCr} 2$ at higher forces than for $\mathrm{CuCr} 25$. The reason behind this is not clear, it is possible that the lower conductivity of chromium leads to higher contact resistance, however, this is not proven.

Due to this information it was possible to start the investigation of the change of contact resistance in dependence of the number of mechanical switching operations. The results show that the resistance of first operation for all materials is much lower than the value after 1000 or 2500 operations. The value of $\mathrm{Cu}$ and $\mathrm{CuCr} 2$ increases nearly by a factor of 100 . $\mathrm{CuCr} 25$ behaves similarly, but starts at a higher resistance. In order to achieve a somehow stable resistance, a number of switching operations are necessary. The measurement series with 2500 mechanical switching operations shows that a relatively stable contact resistance can be reached after a few hundred mechanical switching operations. But as the series \#1 $\mathrm{CuCr} 2$ shows, it is possible that even a value that looks stable can change. The cause for such change is not known. The test procedure was the same for all measurement series. One possible reason could be, that the contacts do not make contact at the exact same position after closing. This is an important finding for the development of a contact system for a UFD. After first operation it seemed that $\mathrm{Cu}$ or $\mathrm{CuCr} 2$ is superior with regard to contact resistance. A small contact resistance leads to less heating of the contacts and therefore increases the current carrying capability. The long measurement series shows that the difference between $\mathrm{CuCr} 25$ and $\mathrm{Cu}$ decreases and after 2500 operations it is only $25 \%$ higher. Until now it is not clear which physical effect causes the change of contact resistance. A first inspection of the surface, after 2500 mechanical switching operations with a confocal microscope (resolution is in the range of micrometer), does not show any significant change. All measurements are carried out under atmospheric conditions. Copper and chromium oxidize and therefore a thin oxide layer will grow at the surface over time. A measurement series with 2500 operations took around $75 \mathrm{~h}$, so they were exposed to air over a significant amount of time. However, it is known [14] that the oxide layer shows a logarithmic growth. After a few minutes most of the oxide layer has already grown. Therefore, even during the first operation, an oxide layer is present. From what is known today, the oxide layer cannot explain the change of resistance during a large number of switching operations.

\section{CONCLUSION}

The conducted measurements show that a single measurement of the contact resistance is not sufficient, if the value should be used in the design process of an UFD. Therefore, the switching behavior is simulated until a steady state resistance is archived. This resistance is for all tested materials signifi- cantly larger than the value of the first operation, which must be considered when dimensioning the heat sink. Moreover, the change of resistance depends on the material, so each material has to be tested. It could be shown that the steady state resistance is for all materials in the same order of magnitude and therefore no material can be excluded for the UFD.

While during these experiments the current was limited to $100 \mathrm{~A}$ to minimize the heating, in a next step the current will be increased to the nominal current $\left(I_{\mathrm{n}} \approx 2000 \mathrm{~A}\right)$ of a UFD. This will lead to a significant heating of the contact and therefore to a faster growth of the oxide layer. In order to separate the effect of heating and the oxide layer, these tests should be carried out in an inert gas atmosphere (e.g. helium or argon).

During the first series it was assumed that the contacts will open with zero current. In a real UFD the residual current is determined by the LCS and the main breaker. Therefore, it is likely that the UFD has to break a small residual current in the range of $10 \mathrm{~mA}$ to $1000 \mathrm{~mA}$, which could also affect the choice of material.

\section{REFERENCES}

[1] Cigré, Compendium of all HVDC projects, 2009

[2] IPFS, List of HVDC projects, https://ipfs.io/ipfs/ QmXoypizjW3WknFiJnKLwHCnL72vedxjQkDDP1mXWo6uco/ wiki/List_of_HVDC_projects.html, visited 2019-04-10.

[3] N. Flourentzou, G. A. Vassilios, D. D. Georgios, "VSC-based HVDC power transmission systems: An overview, IEEE Transactions on power electronics", 2009.

[4] "Technical Requirements and Specifications of State-of-the-Art HVDC Switching Equipment", Cigré Technical Brochure TB 683, 2017.

[5] M. Callavik, A. Blomberg, J. Häfner, B. Jacobson, "The Hybrid HVDC Breaker - An innovation breakthrough enabling reliable HVDC grids", ABB Grid Systems, Technical Paper Nov. 2012.

[6] V. Lenz, T. Schultz, C.M. Franck, "Impact of topology and fault current on dimensioning and performance of HVDC circuit breakers", 4h International Conference of Electric Power Equipment - Switching Technology, 2017.

[7] H. Menne, C.M. Franck, "Contact resistance measurement on contacts for an Ultra Fast Disconnector", VDE High Voltage Conference, November 2018.

[8] E. Vinaricky, K.-H. Schröder, J. Weiser, "Elektrische Kontakte, Werkstoffe und Anwendungen", Springer Vieweg, 3rd edition, 2016.

[9] P.G. Slade, "Electrical Contacts", CRC Press, 2nd edition, 2017.

[10] Plansee, https://www.plansee.com/en/produkte/komponenten/ elektrische-kontakte/kupfer-chrom-cucr.html, visited 2019-04-14.

[11] N. Giao Trinh, "Electrode design for testing in uniform field gaps", IEEE Transactions on Power Apparatus and Systems, June 1980.

[12] IEC 62271-100, "High-voltage switchgear and controlgear - Part 100 Alternating-current circuit-breakers", 2018.

[13] J.J. Shea, J.A. Bindas, "Measuring molded case circuit breaker resistance", Proceedings of the 38th Holm Conference, 1992.

[14] A.H. White, L.H. Germer, "The Rate of Oxidation of Copper at Room Temperature", Journal of Electrochemical Society, 1942. 